\title{
Mineração
}

\section{Planejamento para o fechamento prematuro de minas}

\section{Planning for early mine closure}

\section{Luis Enrique Sánchez}

Professor Titular do Departamento

de Engenharia de Minas e de Petróleo

Escola Politécnica da Universidade

de São Paulo

Isanchez@usp.br

\section{Resumo}

Planos de fechamento de mina são hoje bastante difundidos entre empresas de mineração no Brasil. No entanto, a principais diretrizes brasileiras e internacionais abordam principalmente o fechamento programado de uma mina, ou seja, aquele que ocorre após a exaustão das reservas, segundo um plano de lavra predeterminado. Esse trabalho revê dois casos recentes de fechamento prematuro de mina na Austrália e propõe, com base nas boas práticas internacionalmente recomendadas para o planejamento do fechamento de minas, critérios para avaliar o grau de preparação das empresas para o fechamento prematuro. Os critérios são testados em planos de fechamento de duas minas de ferro.

Palavras-chave: Fechamento de mina, fechamento prematuro, riscos, impactos socioeconômicos.

\begin{abstract}
Several mining companies operating in Brazil have been preparing mine closure plans describing end of life actions to ensure long term physical stability, land rehabilitation and, to a certain extent, socioeconomic measures. Most Brazilian and international guidelines, however, skip early closure and only feature guidance to closure planning after reserve depletion. In this paper, two cases of early closure in Australia are reviewed, causes for early closure are explored, criteria to evaluate preparedness to early mine closure are proposed and tested in two mines. It concludes that, despite recent advances, planning for mine closure seldom take account of early closure risks.
\end{abstract}

Keywords: Mine closure, early closure, risks, social and economic impacts.

\section{Introdução}

Planos de fechamento de mina são preparados por diversas empresas de mineração no Brasil, seja para atender determinações legais - como é o caso do Estado de Minas Gerais -, seja para atender a políticas corporativas - como fazem empresas nacionais e multinacionais -, seja, ainda, para estimar o montante do passivo ambiental para atender a regulamentações do mercado de capitais em bolsas de valores estrangeiras (Sánchez, 2005). O adequado planejamento para o fechamento pode proteger acionistas, governos, fornecedores, comunidades locais e as gerações futuras dos impactos socioeconômicos do fechamento, assim como reduzir o passivo ambiental, recuperar ambientes degradados e resultar em um balanço positivo para a região onde se insere cada mina.

Na mineração, entretanto, todo planejamento envolve incertezas. Há incertezas acerca de volumes e teores de reservas minerais e desconhecimento dos preços futuros das matérias-primas minerais. Durante a primeira década do século XXI, os preços de diversos metais atingiram níveis sem precedentes, o que possibilitou o aproveitamento de depósitos minerais até então marginais ou não econômicos. 
Da mesma forma, a queda das cotações a partir de setembro de 2008 ocasionou o fechamento de diversas minas.

As incertezas inerentes à mineração também afetam o planejamento para o fechamento de minas. Este trabalho discute algumas situações que podem resultar em fechamento prematuro de uma mina e explora critérios que podem ser aplicados para que o plano de fechamento analise de forma adequada o cenário de fechamento prematuro.

\section{Do abandono ao fechamento de minas}

As conseqüências ambientais e socioeconômicas do fechamento de minas vêm sendo objeto de estudos e regulamentação em várias partes do mundo. Durante séculos, as minas foram simplesmente abandonadas, sem que seus efeitos fossem percebidos como merecedores de preocupação. Porém, o acelerado crescimento econômico observado ao longo do século XX levou a um aumento da demanda e, conseqüentemente, da produção mineral superior à taxa de crescimento da população (UNEP, 2010). O abandono, então, deixou de ser uma opção aceitável.

Casos como o da mina de Summitville, nos EUA, atraíram a atenção da opinião pública e impulsionaram a regulamentação. Esta mina de ouro situada no Estado de Colorado foi abandonada em dezembro de 1992 quando a empresa faliu, dias depois de apresentar um plano de recuperação ambiental orçado em 40 milhões de dólares (face a uma garantia financeira de 5 milhões anteriormente apresentada ao governo estadual). Na falta de leis estaduais, a responsabilidade pelo local foi assumida pelo governo federal, por intermédio da Environmental Protection Agency, que colocou o sítio na lista prioritária de áreas contaminadas (Filas \& Gormley, 1997). Dessa forma, a coletividade herdou um passivo ambiental e uma área degradada cuja recuperação ainda não fora concluída até maio de 2010 (USEPA, 2010).

Estudos sobre os danos ambientais ocasionados por minas abandonadas foram realizados em várias partes do mundo. Os inventários nacionais ou regionais do número de tais minas alcançam a cifra de dezenas de milhares (Sánchez, 2001), dependendo dos critérios adotados. Evidentemente, nem todas apresentam o mesmo grau de risco ambiental e muitas apresentam interesse histórico que as pode qualificar como elementos do patrimônio industrial mineiro (Villas-Bôas \& Albuquerque, 2003). Francaviglia (1991, p. xvii) registra o legado da mineração na paisagem: "Onde quer que mineiros tenham trabalhado e minérios tenham sido processados, pode-se ver os resultados do seu labor".

As primeiras respostas do setor mineral à degradação ambiental foram as ações de recuperação ou de reabilitação, hoje plenamente incorporadas à mineração em escala internacional. Marshall (1983) descreveu as mudanças do tratamento da recuperação de áreas degradadas (RAD) na mineração do Canadá, em resposta à evolução das exigências legais. A partir de uma fase inicial em que a recuperação era planejada e executada somente após o encerramento das atividades extrativas, passou-se para uma fase de planejamento ainda durante a operação da mina, mas com a implementação de medidas de recuperação somente depois de terminadas as atividades de lavra, para, em seguida, evoluir para a implementação de medidas de recuperação de modo concomitante ou paralelo à continuidade da lavra. O último estágio corresponde a planejar a reabilitação juntamente com o planejamento da mina.

O objetivo da RAD é normalmente apresentado como o de tornar a área apta para um novo uso, garantindo-se a condição de estabilidade física e química para que o novo uso seja seguro, ou a reconstituição de um ecossistema auto-sustentável. Iniciativas de RAD têm sido bem sucedidas em diversos casos de mineração (Nichols \& Nichols, 2003; Norman et al., 2006; Parrota \& Knowles, 2001; Toy \& Griffith, 2001). Porém, mesmo os melhores exemplos de $\mathrm{RAD}$ não abordam as demais questões associadas ao fechamento de uma mina (Lima et al., 2006). Estas questões envolvem, principalmente, a mitigação dos impactos socioeconômicos decorrentes do fechamento.

No passado, o abandono de uma mina podia significar o abandono de pequenas cidades mineiras (Francaviglia, 1991). Ainda hoje o fechamento pode levar à desativação ou a demolição de alojamentos e vilas residenciais, principalmente em regiões remotas como o norte do Canadá ou o deserto australiano. Em muitos países em desenvolvimento, contudo, contingentes populacionais apreciáveis podem ser atraídos por projetos de mineração, mesmo em regiões remotas e os impactos sociais do fechamento são "exacerbados" (World Bank \& IFC, 2002). Esse é certamente o caso do Brasil, onde os principais impactos socioeconômicos do fechamento de minas incluem:
- Perda de arrecadação tributária, principalmente municipal.

- Perda de empregos e renda.

- Diminuição da atividade econômica local.

- Redução de qualidade e alcance dos serviços públicos.

- Perda de qualidade de vida da população local.

Os impactos sócio-econômicos decorrentes do fechamento de minas não são tratados nos planos de recuperação de áreas degradadas (PRADs) ou outros estudos técnicos de reabilitação. Enquanto os impactos sociais da abertura de uma nova mina são discutidos - nem sempre com a profundidade necessária - nos estudos de impacto ambiental que precedem a licença ambiental de um novo empreendimento, os impactos do fechamento são muitas vezes negligenciados (Taveira, 2003). Um levantamento internacional feito em 2001 mostrou que a maioria das empresas de mineração estudadas dispunha de "planos de mitigação ambiental pós-fechamento", mas somente metade destas empresas tinha planos sócio-econômicos detalhados e periodicamente atualizados (Azapagic, 2004, p. 645).

Durante os anos de 1990 os problemas socioambientais relativos ao fechamento de minas passaram a ser reconhecidos como um novo desafio para as empresas do setor e planejar o fechamento passou a ter como objetivo reduzir os impactos ambientais residuais e mitigar os impactos sócio-econômicos.

Assim, o reconhecimento de que há necessidade de planejar o fechamento de minas advém de três frentes: (i) da constatação dos danos ambientais e dos riscos causados por minas abandonadas ou fechadas sem que tenham sido tomados os necessários cuidados visando à proteção ambiental, (ii) da constatação que a economia e a sociedade de uma região mineira podem ser negativa e significativamente afetadas quando minas fecham e (iii) da constatação de que os PRADs abordam somente parte das questões relevantes associadas ao fechamento de uma mina.

A discussão internacional sobre a desativação de minas deu origem a exigências legais em diversos países (Clark, 2000), as- 
sim como a algumas diretrizes de aplicação internacional para planejar o fechamento, como ANZMEC/MCA (2004), Austrália (2006), Heikkinen et al. (2008), IIED (2002) e ICMM (2008). Sinteticamente, considera-se a necessidade de:

I Prover recursos financeiros para a desativação e para a etapa de pósfechamento.

II Adotar uma estratégia de aproximações sucessivas, em que o fechamento é planejado em concordância com o plano de lavra e revisado periodicamente ou quando

\section{Fechamento prematuro}

Fechamento prematuro é aqui definido como a cessação das atividades mineiras antes do previsto no plano de lavra. As causas do fechamento prematuro podem ser:

(1) Queda dos preços das matérias primas minerais.

(2) Redução do mercado para determinados bens minerais por razões de saúde (como o amianto) ou por competição com outros materiais.

(3) Acidentes ou incidentes de operação, como rupturas de barragens de rejeitos ou desmoronamento de escavações subterrâneas.

(4) Decisões empresariais decorrentes de venda de ativos, fusões ou aquisições ou mudança de composição acionária.

(5) Eventos externos extremos decorrentes de processos geológicos, atmosféricos ou mudanças climáticas.

(6) Mudanças de políticas governamentais, como aumento de impostos, decisões administrativas motivadas por pressão da comunidade ou decisões judiciais.

(7) Conhecimento geológico insuficiente acerca da jazida.

(8) Erros de projeto que causem dificuldades operacionais ou custos elevados.

(9) Fraude ou outras práticas comer- de mudanças no plano de lavra.

III Que as medidas a serem implementadas durante a fase de desativação sejam previstas desde o primeiro planejamento da mina.

IV Minimizar tanto os impactos físico-ecológicos quanto os sócio-econômicos.

Em Minas Gerais, único Estado a dispor de regulamentação sobre o tema (Deliberação Normativa COPAM No 127 , de 27 de novembro de 2008), um plano de fechamento é exigido dois anos antes da data programada para fechamento. A regulamentação também prevê os casos de paralisação temporária, mas neste caso a empresa deve apenas comunicar o fato e apresentar uma relação de medidas de proteção até 180 dias após a paralisação. A Norma Regulamentadora da Mineração 20, do Departamento Nacional da Produção Mineral, prevê os casos de suspensão das operações mineiras (cessação temporária das atividades) e de fechamento (cessação definitiva das operações mineiras), mas também demandando a comunicação mediante justificativa. ciais ilícitas.

Como se vê, algumas causas são passíveis de controle pela empresa, ao passo que outras dependem de fatores além de sua capacidade de influência. Todavia, independentemente da sua capacidade de controle, as empresas devem estar preparadas para a eventualidade do fechamento prematuro, uma vez que suas conseqüências ambientais e socioeconômicas são pelo menos tão importantes quanto, e possivelmente mais críticas do que aquelas decorrentes do fechamento programado.

Laurence (2006) exemplifica minas que fecharam por diversos motivos. $\mathrm{O}$ fechamento prematuro pode ser mais comum do que o normalmente admitido. Laurence (2010) examinou cerca de mil casos de fechamentos de minas, a maioria na Austrália, no período 19812009 , observando que cerca de $75 \%$ deles ocorreram antes da exaustão das reservas. Bentel (2009) adverte que "o fechamento súbito ou prematuro (...) pode acarretar em aumento dramático dos custos”.

As boas práticas internacionalmente recomendadas para o planejamento do fechamento de minas abordam, basicamente, o fechamento programado, ou seja, aquele que ocorre de acordo com a vida útil da mina prevista em seu plano de lavra. Como o plano de lavra é usualmente modificado e atualizado durante a fase de operação de uma mina, essas alterações devem também resultar em revisão do plano de fechamento. O principal guia internacional da atualidade (ICMM, 2008), é todo voltado para o fechamento programado, ainda que mencione brevemente a situação de "fechamento súbito" (termo usado na edição brasileira, equivalente ao original sudden closure), a qual não corresponde plenamente ao fechamento prematuro, que não é necessariamente súbito.

Defende-se aqui que tanto o fechamento programado quanto o prematuro devem ser considerados na elaboração de planos de fechamento. Ademais, deve também ser considerada uma etapa intermediária de suspensão temporária das atividades (também descrita na literatura como de cuidado e manutenção), que pode conduzir à reabertura, ao fechamento definitivo ou à venda da mina para outra empresa. A Figura 1 ilustra esses cenários com a ajuda de uma linha do tempo. Existe ainda a possibilidade de reabertura pós-fechamento, anos mais tarde, mas este cenário pode ser adequadamente tratado como um novo projeto sujeito a licencia-

Figura 1

Principais etapas do período de vida de uma mina.

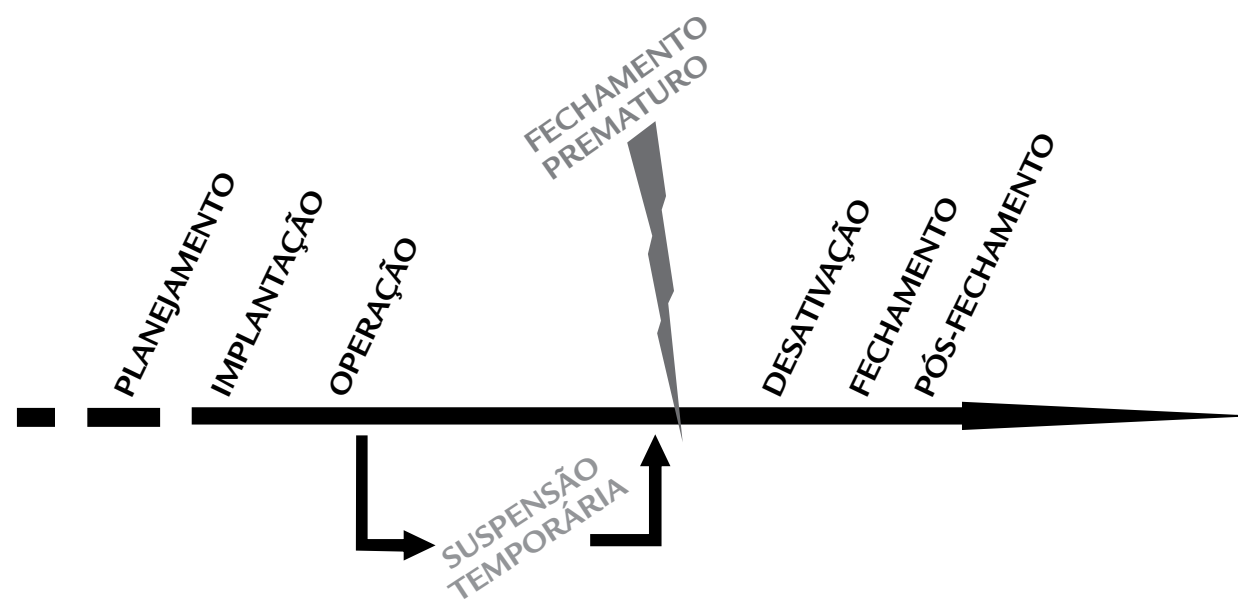


mento e avaliação prévia de impactos.

Há dificuldades adicionais na preparação para o fechamento prematuro, como o curto período para implementação de programas de desativação, a necessidade

\section{Mina Beenup}

Essa mina de areias pesadas, localizada cerca de $300 \mathrm{~km}$ ao sul de Perth, no sudoeste da Austrália, começou a ser implantada em 1995, entrou em funcionamento em janeiro de 1997 e foi fechada em fevereiro de 1999. Desde então, encontra-se em fase de pós-fechamento, com atividades de reabilitação e monitoramento ambiental. Trata-se de um caso de fechamento prematuro que requereu uma revisão completa do plano de fechamento preparado durante a fase de planejamento da mina.

O plano de lavra previa a extração do minério por dragas flutuantes de grande porte, escavando painéis com 40m de profundidade. O minério era concentrado em espirais classificadoras e o rejeito lançado em painéis exauridos, técnica utilizada com sucesso em outras minas da empresa. Para armazenar o rejeito oriundo do tratamento do minério extraído do primeiro painel foi construído um dique circular para conformar a bacia de rejeito. No entanto, situações inesperadas como a alta abrasividade do minério, que aumentou o custo de beneficiamento, e a baixa taxa

\section{Mina Ravensthorpe}

A crise econômica que teve início em setembro de 2008 afetou severamente os preços das matérias-primas. Alguns metais, em particular, tiveram sua cotação internacional bastante reduzida. Em conseqüência, minas fecharam em várias partes do mundo. Uma delas foi a mina Ravensthorpe, situada no sudoeste da Austrália, que teve suas atividades suspensas em janeiro de 2009, menos de um ano depois de ter sido aberta (maio de 2008). Depois de um investimento de 2,2 bilhões de dólares australianos, a empresa $\mathrm{BHP}$ vendeu a mina de níquel laterítico por 340 milhões para a empresa canadense

\section{Liçoes aprendidas}

Os inúmeros casos de minas abandonadas - e particularmente aqueles mais graves como o da mina Summitville - chamaram a atenção para a necessidade de prevenir novas ocorrências. A de se adiantarem medidas de reabilitação e os problemas socioeconômicos para a comunidade local. O fechamento prematuro de uma mina sem que a empresa esteja devidamente preparada pode trazer

de sedimentação dos finos dispostos nos painéis lavrados, que levou à necessidade de construção de diques adicionais para armazenamento de rejeitos, levaram à decisão de fechar a mina apenas dois anos depois de sua abertura, face a uma vida útil prevista de vinte anos. O custo do fechamento foi estimado em A $\$ 44$ milhões, ao passo que o investimento na mina foi da ordem de A\$ 250 milhões.

O projeto de fechamento incluiu ações de recuperação de ecossistemas e controle de drenagem ácida. Antes da abertura da mina, a empresa BHP havia criado um grupo consultivo comunitário, convidando representantes de diferentes segmentos sociais, para debater as principais questões suscitadas pelo projeto. O plano de reabilitação foi preparado mediante consulta à comunidade, aproveitando a existência desse grupo consultivo. Dentre as opções de uso do solo avaliadas, decidiu-se que cerca de $80 \%$ da área seria dedicada ao restabelecimento de vegetação nativa e áreas alagadas (wetlands) e 20\% seria usada como pastagem. A área total afetada pela mina e instalações auxiliares

First Quantum Minerals, que assumiu o controle do sítio em fevereiro de 2010, programando o reinício das atividades para 18 meses depois dessa data.

Tecnicamente a mina Ravensthorpe está com suspensão temporária por período indeterminado (ou cuidado e manutenção), o que significa que a empresa continua responsável pelo sítio, executando ações de manutenção e garantindo a segurança. A venda da mina significa que a nova proprietária assume essa responsabilidade. Porém, os impactos socioeconômicos independem. Dos 650 postos de trabalho, a BHP recolocou 110 consequências negativas para a comunidade, para o ambiente e para a própria empresa. Na sequência, dois casos recentes de fechamento prematuro serão brevemente revistos. é de cerca de 340 hectares. Essa opção de uso do solo implicou a remoção de cerca de 2,5 milhões de toneladas de areia (estéril de mina) de pilhas de estéril e sua recolocação em áreas designadas para fins de reabilitação.

As metas de reabilitação (completion criteria) foram propostas em forma de minuta pela empresa ao governo em 2004, revisados após análise dos departamentos competentes, discutidos com o grupo consultivo e submetidos em forma final em novembro de 2006. No início de 2010 ainda aguardam aprovação formal. Há 28 metas a serem atingidas, relativas a (1) qualidade das águas superficiais, (2) estabilidade da estrutura de retenção de rejeitos, (3) controle de erosão, (4) diversidade de ecossistemas, (5) diversidade florística, (6) resiliência dos ecossistemas e (7) uso do solo (BHP Billiton, 2009). Não há metas de natureza social ou econômica. Os cerca de cem empregos permanentes da mina foram absorvidos por outras atividades. A influência do empreendimento nas finanças municipais era mínima, uma vez que não há impostos locais incidentes. em outras minas (Spencer, 2009a), ao passo que os novos empreendimentos comerciais instalados na região para atender à mina foram severamente afetados, da mesma forma que o mercado imobiliário (Spencer, 2009b).

A mina tinha vida útil prevista de 25 anos (Perpitch, 2009) e atraiu diversos investimentos privados em pequenos negócios como restaurantes, farmácias, loteamentos e construção civil, em parte apoiados pela própria empresa. Mesmo uma possível reabertura, mais de dois anos após a suspensão das atividades, pode não ser mitigação suficiente para as perdas. recuperação de áreas degradadas é uma resposta a essa necessidade, assim como a obrigatoriedade de apresentação de garantias financeiras por parte das empresas de mineração e a necessidade de incorporar os custos de fechamento aos balanços e demonstrações financeiras de empresas de capital aberto. O planejamento para o fechamento é uma evolução no tratamento da questão, quando se passa a 
considerar o planejamento ambiental de todo o ciclo de vida de uma mina, desde sua concepção até a transferência da área para outra forma de uso pós-mineração (Sánchez, 2004). A incorporação desse conceito ao planejamento de novas minas tem o potencial de contribuir de maneira significativa para mudar a imagem da mineração de um agente de degradação para a de um promotor do desenvolvimento sustentável, como sugere o extenso e intenso trabalho com perspectiva de longo prazo desenvolvido para a nova mina de bauxita de Juruti (GVCES, Alcoa e Funbio, 2008).

No entanto, em que pesem tais avanços, um adequado planejamento deve necessariamente considerar os riscos de fechamento prematuro, cenário que pa- rece não ter sido ainda satisfatoriamente incorporado nem mesmo aos melhores casos das empresas mais avançadas.

Minas de minerais metálicos são mais vulneráveis a flutuações de preços e, portanto, mais expostas ao risco de fechamento prematuro. Talvez o caso mais dramático seja o do ouro, pois um período longo de preços elevados e mesmo crescentes, tem levado à abertura de muitas novas minas ou à reabertura de minas já paralisadas, iniciando operações de custos elevados que somente são cobertos por preços altos do metal. Naturalmente, essas minas podem fechar se houver uma queda sustentada das cotações internacionais. Raciocínio similar se aplica a outros metais, principalmente aqueles cujos preços são formados por contratos de curto prazo. Mesmo o minério de ferro, cuja formação de preços no mercado internacional tem historicamente se dado por negociações diretas entre grandes produtores e grandes compradores, tem passado por transformações que podem levar a uma maior volatilidade dos preços.

Por outro lado, as frequentes transações de compra e venda de ativos entre empresas reforçam a necessidade de planejamento para fechamento prematuro, na medida em que estas operações podem levar a mudanças de gestão e a diferentes políticas corporativas que podem por em xeque as relações entre uma mina e a comunidade anfitiriã. Nestes casos, a existência de um planejamento sólido pode reduzir os impactos de mudanças súbitas de postura da parte das empresas.

\section{Avaliação do grau de preparação das empresas}

Diferentes stakeholders têm interesse em minimizar os riscos do fechamento prematuro de uma mina. Para acionistas e investidores, o fechamento prematuro pode representar perda de valor de ativos. Para as empresas, riscos de imagem. Para os trabalhadores e a comunidade local, perda de empregos e renda. Para os fornecedores e prestadores de serviço, perdas econômicas e de postos de trabalho. Para os governos, perda de arrecadação tributária e, eventualmente, custos de reparação de danos ambientais. Para a sociedade e as gerações futuras, o fechamento prematuro pode representar danos ambientais de longa duração.

Dessa forma, é conveniente que se possa avaliar em que medida as empresas de mineração estão preparadas para fazer face aos riscos de fechamento prematuro. Embora os principais guias de boas práticas para o planejamento do fechamento de minas (ANZMEC/MCA, 2000; Australia, 2006; Heikkinen et al., 2008; ICMM, 2008) estipulem a necessidade de considerar o cenário de fechamento prematuro, não o exploram com o mesmo detalhe dado ao cenário de fechamento programado.

Assim, são aqui propostos, com base em adaptação de proposições obtidas na literatura e nas boas práticas internacionalmente recomendadas para planejamento do fechamento de minas, os seguintes critérios para avaliar o grau de preparação das empresas para o fechamento prematuro:

(1) O plano de fechamento deve contemplar o cenário de fechamento prematuro.
(2) O plano de fechamento deve identificar as possíveis causas de fechamento prematuro pertinentes à mina.

(3) O plano de fechamento deve descrever com suficiente detalhe as ações necessárias em caso de fechamento, especificando as responsabilidades das partes.

(4) O plano de fechamento deve incluir, para o cenário de fechamento prematuro, ações pós-fechamento de monitoramento, manutenção e possíveis compensações sociais.

(5) As ações previstas no plano de fechamento devem ser baseadas em conhecimento sólido acerca do ambiente biofísico e socioeconômico da mina e seu entorno; para tal, estudos de detalhe sobre aspectos como hidrogeologia, geotecnia, recuperação de áreas degradadas e outros temas pertinentes devem ter sido realizados e mantidos atualizados.

(6) O risco de fechamento prematuro deve ser avaliado e comunicado às partes interessadas.

(7) Os custos das ações previstas no cenário de fechamento prematuro devem ser estimados.

(8) A empresa deve fazer provisões financeiras ou fornecer garantias suficientes para cobrir os custos do fechamento prematuro.

(9) As partes interessadas e, em especial, a comunidade local, devem ter sido consultadas quanto as ações a serem implementadas em caso de fechamento prematuro.

(10) A empresa deve desenvolver um conjunto de indicadores apropriados que possibilitem demonstrar, ao fim do processo, que os objetivos do fechamento tenham sido atingidos.

(11) O plano de fechamento deve ser revisto e atualizado periodicamente.

$\mathrm{O}$ atendimento a estes critérios pode ser verificado pelas próprias empresas, com relação aos planos existentes. Os critérios também podem ser usados por diferentes partes interessadas. A aplicação dos critérios propostos para avaliar o grau de preparação de uma empresa para o evento de fechamento prematuro não pode se restringir à análise do plano de fechamento e de outros documentos, e deve incluir a coleta de outras evidências de aderência aos critérios, por meio de inspeções técnicas, entrevistas com pessoas responsáveis na empresa e com representantes de stakeholders relevantes e outros procedimentos apropriados. Uma empresa satisfatoriamente preparada para o fechamento prematuro de uma mina provavelmente estará também adequadamente preparada para o fechamento programado.

A título de teste, e para fins exploratórios, os critérios foram aplicados a duas minas de ferro em operação, uma situada no Brasil (caso 1) e outra na Austrália (caso 2), cujas principais características são mostradas no Tabela 1 . A análise expedita mostrou que não existem diferenças sensíveis entre ambos os casos, em que o fechamento prematuro não é explicitamente tratado nos respectivos planos de fechamento (Tabela 2).

Foi adotada uma classificação dicotômica para verificar o atendimento aos critérios, equivalente ao procedimento adotado em estudos qualitativos sobre 


\begin{tabular}{c|c|c}
\hline Características & Caso 1 & Caso 2 \\
\hline $\begin{array}{c}\text { Principais razões } \\
\text { de fechamento }\end{array}$ & $\begin{array}{c}\text { 1. Diretriz corporativa. } \\
\text { 2. Atendimento a requisitos de órgão } \\
\text { regulador do mercado de capitais. }\end{array}$ & $\begin{array}{c}\text { 1. Diretriz corporativa. } \\
\text { 2. Atendimento a requisitos de órgão regulador } \\
\text { do mercado de capitais. }\end{array}$ \\
\hline $\begin{array}{c}\text { Ano de elaboração do } \\
\text { plano de fechamento }\end{array}$ & 2007 & $\begin{array}{c}2008 \\
\text { ambiental para para fins de licenciamento. }\end{array}$ \\
\hline $\begin{array}{c}\text { elaborado o plano de } \\
\text { fechamento }\end{array}$ & Operação a plena capacidade. & $\begin{array}{c}\text { Projeto de expansão que aumenta a escala de } \\
\text { produção e reduz a vida útil. }\end{array}$ \\
\hline $\begin{array}{c}\text { Vida útil no momento } \\
\text { da elaboração do plano }\end{array}$ & 26 anos & 13 anos \\
\hline Escala de produção & $>45 \mathrm{Mt}$ & $45 \mathrm{Mt} /$ ano \\
\hline
\end{tabular}

conteúdo e abrangência de relatórios de sustentabilidade de empresas de mineração (Pérez \& Sánchez, 2009). Quando o primeiro critério for atendido (o que não ocorreu com os dois casos estudados), o método de análise deve ser refinado, por exemplo, por meio da utilização de escalas qualitativas para cada critério, como o procedimento de avaliação de práticas de recuperação de áreas degradadas desenvolvido por Neri e Sánchez (2010).

Os resultados sintetizados na Tabela 2 indicam que o cenário de fechamento prematuro não é explicitamente considerado nos planos de fechamento. Porém, sabe-se que os custos desse cenário são estimados pelas empresas, reportados internamente e, de forma consolidada, transmitidos às autoridades de regulação dos mercados de valores mobiliários dos EUA e de outros países que exigem a informação sobre custos de remoção de ativos, obrigando também as empresas a fazerem provisões contábeis para tal (Sánchez, 2005).

Em ambos os casos, é informado

\begin{tabular}{|c|c|c|}
\hline Critérios de planejamento para fechamento prematuro & Caso 1 & Caso 2 \\
\hline 1. O plano de fechamento deve contemplar o cenário de fechamento prematuro. & não & não \\
\hline $\begin{array}{l}\text { 2. O plano de fechamento deve identificar as possíveis causas de fechamento prematuro } \\
\text { pertinentes à mina. }\end{array}$ & não & não \\
\hline $\begin{array}{l}\text { 3. O plano de fechamento deve descrever com suficiente detalhe as ações necessárias } \\
\text { em caso de fechamento prematuro, especificando as responsabilidades das partes. }\end{array}$ & não & não \\
\hline $\begin{array}{l}\text { 4. O plano de fechamento deve incluir, para o cenário de fechamento prematuro, ações } \\
\text { pós-fechamento de monitoramento, manutenção e possíveis compensações sociais. }\end{array}$ & não & não \\
\hline $\begin{array}{l}\text { 5. As ações previstas no plano de fechamento devem ser baseadas em conhecimento } \\
\text { sólido acerca do ambiente biofísico e socioeconômico da mina e seu entorno. }\end{array}$ & $\operatorname{sim}$ & $\operatorname{sim}$ \\
\hline $\begin{array}{l}\text { 6. Os riscos associados ao fechamento prematuro deve ser avaliado e comunicado às } \\
\text { partes interessadas. }\end{array}$ & não & não \\
\hline $\begin{array}{l}\text { 7. Os custos das ações previstas no cenário de fechamento prematuro devem ser } \\
\text { estimados. }\end{array}$ & $\operatorname{sim}$ & $\operatorname{sim}$ \\
\hline os custos do fechamento prematuro. & $\operatorname{sim}$ & $\operatorname{sim}$ \\
\hline $\begin{array}{l}\text { 9. As partes interessadas e, em especial, a comunidade local, devem ter sido consultadas } \\
\text { quanto as ações a serem implementadas em caso de fechamento prematuro. }\end{array}$ & não & $\operatorname{sim}$ \\
\hline $\begin{array}{l}\text { 10. A empresa deve desenvolver um conjunto de indicadores apropriados que possibilitem } \\
\text { demonstrar, ao fim do processo, que os objetivos do fechamento tenham sido atingidos. }\end{array}$ & não & não \\
\hline 11. O plano de fechamento deve ser revisto e atualizado periodicamente. & $\operatorname{sim}$ & $\operatorname{sim}$ \\
\hline
\end{tabular}

Notas:

(a) as perguntas (4) (5) (6) (7) (8) foram respondidas considerando o cenário de fechamento programado, uma vez que se aplicam a ambos os casos.

(b) para a pergunta (11) considerou-se se o plano informa haver previsão de revisão e atualização.

\section{Conclusões}

Em que pese o destaque que o tema fechamento de minas tem recebido no setor de mineração no Brasil, os riscos de fechamento prematuro ainda não são reconhecidos.

$\mathrm{Na}$ regulamentação de Minas Ge- rais, não há dispositivo específico sobre fechamento prematuro e as normas regulamentadoras do DNPM tampouco abordam explicitamente este cenário.

Por outro lado, a prática das empresas de planejar o fechamento parece se
Tabela 1

Características básicas

dos casos analisados.

que há previsão de revisão do plano de fechamento, mas somente no caso australiano há explicitação de consulta realizada às partes interessadas, constituindo-se na única diferença notável entre os dois casos. Nenhuma das empresas adota indicadores específicos e locais para acompanhar os efeitos do fechamento ou a eficácia das ações de controle, embora ambas preparem relatórios de sustentabilidade em conformidade com as diretrizes Global Reporting Initiative (Perez \& Sánchez, 2009).

Tabela 2

Ensaio de aplicação dos critérios a duas minas de ferro. dirigir no mesmo sentido de considerar somente o cenário de fechamento programado.

Trata-se de uma fronteira ainda pouco explorada da engenharia de minas e que precisa ser mais bem conhecida. 
ANZMEC/MCA, Australian and New Zealand Minerals and Energy Council/Minerals Council of Australia. Strategic framework for mine closure. Canberra: ANZMEC/ MCA, 2004.

AUSTRALIA. Mine closure and completion. Department of Industry, Tourism and Resources, Canberra, 2006.

AZAPAGIC, A. Developing a framework for sustainable development indicators for the mining and minerals industry. Journal of Cleaner Production, v. 12, p. 639-882, 2004.

BENTEL, G. Key closure planning consideration. In: FOURIE, A., TIBBET, M. (orgs.). Mine Closure 2009, Perth: Australian Center for Geomechanics, 2009, p. 41-54.

BHP Billiton Beenup Rehabilitation Project. Annual Environmental Report 2008-2009.

CLARK, A.L. et al. Legal Framework for Mine Closure. In: KHANNA, T. (org.), Mine closure and sustainable development. London: Mining Journal Books, 2000, p. 93-115.

FILAS, B.A., GORMLEY, J.T. The summitville mine: build-up to crisis. In: MARCUS, J.J. (org.), Mining Environmental Handbook. London: Imperial College Press, 1997, p. 687-697.

FRANCAVIGLIA, R.V. Hard places: reading the landscape of America's mining districts. Iowa City: University of Iowa Press, 1991.

GVCES, Centro de Estudos de Sustentabilidade da Fundação Getúlio Vargas, Alcoa e Funbio, Fundo Brasileiro para a Diversidade. Juruti sustentável: uma proposta para o desenvolvimento local. 2008.

HEIKINNEN, P.M. et al. Mine Closure Handbook. Espoo: Geological Survey of Finland, 2008.

ICMM, Conselho Internacional de Mineração e Metais. Planejamento para o fechamento integrado de mina: kit de ferramentas. London: ICMM, 2008. Publicado em português pelo Ibram, Instituto Brasileiro de Mineração.

LAURENCE, D. Optimisation of the mine closure process. Journal of Cleaner Production, v. 14, p. 285-298, 2006.

LAURENCE, D. Establishing a sustainable mining operation: an overview. Journal of Cleaner Production, no prelo, 2010. DOI 10.1016/j.jclepro.2010.08.019.

LIMA, H.M. et al. Plano de recuperação de áreas degradadas versus plano de fechamento de mina: um estudo comparativo. REM - Revista Escola de Minas, v. 59, n. 4, p. $397-$ 402, 2006.

MARSHALL, I.M. Mining, land use and the environment: II. A review of mine reclamation activities in Canada. Ottawa: Environment Canada, 1983.

NERI, A.C., SÁNCHEZ, L.E. A eficácia das medidas de recuperação ambiental implantadas em minas de calcário para cimento. REM - Revista Escola de Minas, v. 63, n. 2, p. 371-378, 2010.

NICHOLS, O.G., NICLHOLS, F.M. Long-term trends in faunal recolonization after bauxite mining in the Jarrah forest of Southwestern Australia. Restoration Ecology, v. 11, n. 3, p. 261-272, 2003.

NORMAN, M.A. et al. Vegetation succession after bauxite mining in Western Australia. Restoration Ecology, v. 14, n. 2, p. 278-288, 2006.

PARROTTA, J.A., KNOWLES, O. Restoring tropical forests on lands mined for bauxite: examples from the Brazilian Amazon. Ecological Engineering, v.17, p. 219-239, 2001.

PEREZ, F., SÁNCHEZ, L.E. The evolution of sustainability reporting in the mining sector. Environmental Management, v. 43, n. 6, p. 946-961, 2009.

PERPITH, N. Residents want BHP aid after mine shutdown. The West Australian, 24 de janeiro de 2009.

SÁNCHEZ, L.E. Desengenharia: o passivo ambiental na desativação de empreendimentos industriais. São Paulo: Edusp, 2001, 254 p.

SÁNCHEZ, L.E. Planejamento do ciclo de vida de uma mina e redução dos riscos ambientais. In: SIMP. LATINO-AMER ENG MINAS, 1. Anais... São Paulo: Epusp, 2004, p. 347-352.

SÁNCHEZ, L.E. Danos e passivo ambiental. In: PHILIPPI Jr. A. ALVES, A. C. (org.), Curso Interdisciplinar de Direito Ambiental, Barueri, Manole, 2005, p. 261-293.

SPENCER, B. BHP finds jobs for only 110 sacked from Ravensthorpe. The West Australian, 22 de junho de 2009.

SPENCER, B. Mine closure blamed for \$5 M loss. The West Australian, 17 de fevereiro de 2009. 
TAVEIRA, A.L.S. Provisão de recursos financeiros para o fechamento de minas. Escola Politécnica da Universidade de São Paulo, 2003. (Tese de doutorado).

TOY, T., GRIFFITH, J.J. Changing Surface-Mine Reclamation Practices in Minas Gerais, Brazil. International Journal of Surface Mining, Reclamation and Environment, v. 15, n. 1, p. 33-51, 2001.

UNEP, United Nations Environment Program. UNEP Yearbook 2010. Nairobi, 2010.

USEPA, United States Environmental Protection Agency. Superfund Program, Summitville Mine. http://www.epa.gov/region8/superfund/co/summitville/ acesso em 4 de maio de 2010.

VILLAS-BÔAS, A.C., ALBUQUERQUE, G.A.S.C. Património geológico y minero en el contexto del cierre de minas. Rio de Janeiro: CNPq/Cyted, 2003.

WORLD BANK, IFC, International Finance Corporation. It is not over when it is over: mine closure around the world. Washington, 2002.

Artigo recebido em 18 de agosto de 2010. Aprovado em 22 de novembro de 2010. 